O2017 IEEE. Personal use of this material is permitted. Permission from IEEE must be obtained for all other uses, in any current or future media, including reprinting/republishing this material for advertising or promotional purposes, creating new collective works, for resale or redistribution to servers or lists, or reuse of any copyrighted component of this work in other works. This is the author's version of an article that has been published in the conference proceedings. 


\title{
Simultaneous Localization and Mapping for Pedestrians using Low-Cost Ultra-Wideband System and Gyroscope
}

\author{
Christian Gentner, Markus Ulmschneider \\ German Aerospace Center (DLR) \\ Institute of Communications and Navigation \\ Oberpfaffenhofen, 82234 Wessling, Germany \\ Email: \{Christian.Gentner, Markus.Ulmschneider\}@dlr.de
}

\begin{abstract}
Ultra-wideband (UWB) is a promising positioning system that has undergone massive research development in recent years. Most UWB systems assume prior knowledge on the positions of the UWB anchors. Without knowing the anchor positions, an accurate position estimate of a user is difficult. Hence, this paper presents a novel simultaneous localization and mapping (SLAM) approach for pedestrian localization using a UWB system, where the locations of the anchors are unknown. We fuse the distance estimates of the UWB system with heading information obtained from an inertial measurement unit (IMU). We evaluate the proposed algorithm based on measurements with a moving pedestrian and fixed anchors with unknown positions. The evaluations show that an accurate position estimation of both the pedestrian and the anchors is possible without any prior knowledge on the anchor positions.
\end{abstract}

\section{INTRODUCTION}

The proliferation of smartphones has made positioning technologies available to a wide range of users [1]. For outdoor localization, global navigation satellite systems (GNSSs) are the most well-known and mostly used technologies for positioning. In open sky conditions, GNSSs provide a sufficient position accuracy for most mass market applications. However, indoors or in urban canyons the GNSS positioning accuracy might be drastically reduced. In these situations, the received GNSS signals might be blocked, affected by multipath effects or received with low power. To enhance the positioning performance, different methods and sensor systems can provide position information to support or replace GNSSs [2], [3]. Most of the indoor positioning systems use local infrastructure like positioning with radio frequency identification (RFID) [4], mobile communication base-stations [5], [6], wireless local area network (WLAN) [7] or ultra-wideband (UWB) systems [8]-[11]. Using WLAN for indoor positioning is a common approach because WLAN infrastructure is widely deployed [12], [13]. On the other hand, UWB is a promising positioning system that has undergone massive research development in recent years [14], [15]. The localization with UWB signals is based on the parameters extracted from the signals that travel among different nodes. UWB systems use a large bandwidth which enables positioning precision in the order of $10 \mathrm{~cm}$. However, also UWB systems experience multipath and non- line-of-sight (NLoS) propagation which degrade the accuracy significantly. In order to improve the position performance, e.g. [16], [17] use additionally information from an inertial measurement unit (IMU). However, all of the UWB systems assume the prior knowledge on the positions of the UWB base stations, called anchors. Without knowing the anchor positions, an accurate position estimate of the pedestrian is difficult [18].

Contrarily to predetermine the position of the anchors, simultaneous estimation of the receiver and the anchor positions, called simultaneous localization and mapping (SLAM), is attracting a lot of research interest. Generally, SLAM addresses the problem of estimating the location of a moving object and simultaneously building the map of the unknown environment [19]-[23]. In SLAM, the map and the user trajectory are estimated without prior knowledge on the anchor locations. SLAM was originally presented within the robotics community as a technique to jointly estimate the position of a robot and the map of detected landmarks. The most wellknown algorithmic implementations are based on the extended Kalman filter (EKF) [19]-[21], [24], GraphSLAM [25] and FastSLAM [26]. State-of-the art techniques of SLAM within the robotics community use cameras [27] and range measurements [28], [29] to obtain observations of the landmarks. In recent years, pedestrian SLAM became a famous research area [18], [30], [31]. Especially, [18] proposes an indoor pedestrian localization scheme applying SLAM, where ranges from IEEE802.15.4a anchors are measured, and the pedestrian is equipped with an IMU.

In this paper, we present a novel approach which estimates the location of the tag and the receiver simultaneously. We use DecaWave's DW1000 UWB transceiver which enables cost effective real-time positioning with high accuracy in the order of $10 \mathrm{~cm}$ in indoor and outdoor scenarios. In order to estimate the distance between the anchors and receiver (tag), we use a two way ranging method. The proposed SLAM algorithm allows to set up the anchors at arbitrary positions, which are estimated during run-time. To resolve ambiguities, we fuse the distance estimates of the UWB systems with heading information obtained from an IMU. Theoretically, the measurements of the IMU can be directly used in an inertial 


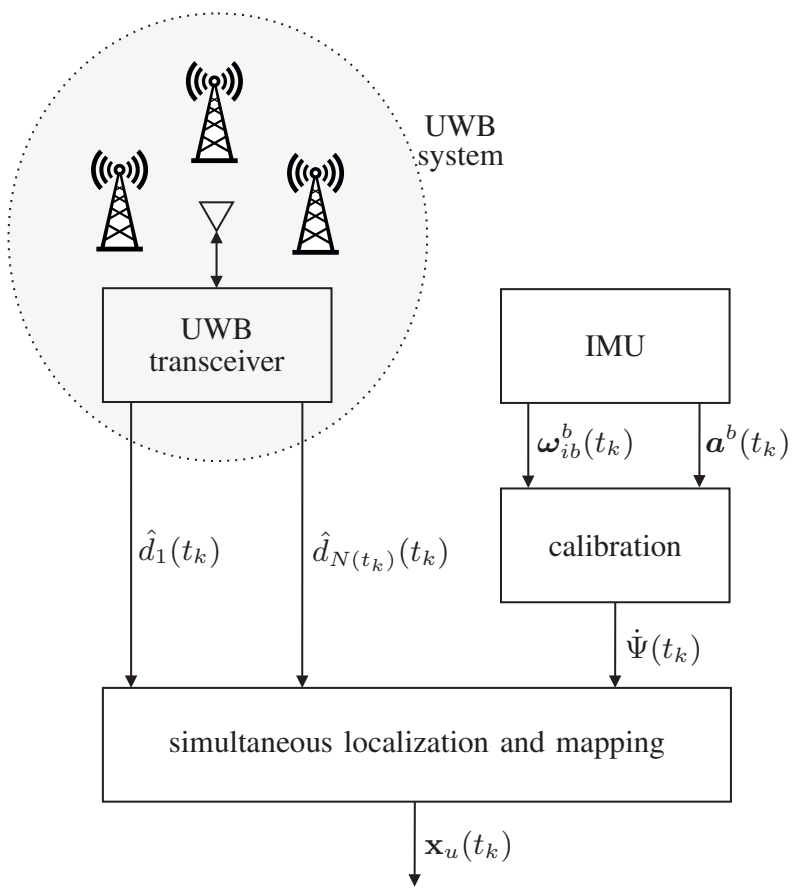

Fig. 1: System model consisting of a UWB receiver and an IMU.

navigation system. However, the position calculation involves double integrations, and hence, even small measurement errors quickly cause a drift in the position solution [32]. To avoid that, we only use heading measurements from the IMU which solely requires an alignment of the coordinate systems. As we are dealing with a relative positioning system, the derived algorithm requires prior knowledge of the initial receiver position and moving direction only to define a local coordinate system. The positioning algorithm derived in this paper is implemented as a Rao-Blackwellized particle filter (RBPF), where a movement model for pedestrians incorporates the heading information from the IMU.

The paper is structured as follows. In Section II, we derive the proposed algorithm. Section III describes the measurement equipment and the indoor measurement scenario. Our algorithm is evaluated based on measurements in Section IV. Finally, Section V concludes the paper.

Throughout the paper, we will use the following notation:

- $(\cdot)^{T}$ stands for matrix (or vector) transpose.

- All vectors are interpreted as column vectors.

- Matrices are denoted by bold capital letters and vectors by bold small letters.

- $\|\mathbf{A}\|^{2}=\sum_{l} \sum_{m}\left|A_{l, m}\right|^{2}$ represents the square of the Frobenius norm of $\mathbf{A}$ with elements $A_{l, m}$.

- $a \sim \mathcal{N}\left(x ; \mu_{a}, \sigma_{a}^{2}\right)$ denotes a Gaussian distributed random variable $a$ with mean $\mu_{a}$ and variance $\sigma_{a}^{2}$.

- $1: k$ stands for all integer numbers starting from 1 to $k$, thus $1,2, \ldots, k$.

- $\mathrm{p}(x)$ denotes the probability density function of $x$.

- $c$ is the speed of light.

\section{Position Estimation}

Fig. 1 presents the available sensors together with the corresponding measurements. As shown on the left, we use an UWB system which estimates the propagation distances

$$
d_{i}\left(t_{k}\right)=\left\|\boldsymbol{r}_{u}\left(t_{k}\right)-\boldsymbol{r}_{\mathrm{A}, i}\left(t_{k}\right)\right\|
$$

between the tag located at $\boldsymbol{r}_{u}\left(t_{k}\right)$ and $N\left(t_{k}\right)$ anchors located at $\boldsymbol{r}_{\mathrm{A}, i}\left(t_{k}\right)$ with $i=1, \ldots, N\left(t_{k}\right)$. We consider a static environment with a fixed number of anchors and a receiver moving along an arbitrary trajectory. However, for notational convenience, a time dependence on $t_{k}$ is introduced here for the anchor positions $\boldsymbol{r}_{\mathrm{A}, i}\left(t_{k}\right)$. The estimated distances $\hat{d}_{i}\left(t_{k}\right)$ of all $N\left(t_{k}\right)$ anchors are used as measurements

$$
\mathbf{z}\left(t_{k}\right)=\left[\hat{d}_{1}\left(t_{k}\right), \ldots, \hat{d}_{N\left(t_{k}\right)}\left(t_{k}\right)\right]^{T}
$$

in the localization algorithm with the corresponding variances $\boldsymbol{\sigma}_{z}\left(t_{k}\right)$.

As mentioned in the introduction, the anchor positions are assumed to be unknown. In order to use the distances estimated by the UWB system, the tag and anchor positions have to be estimated simultaneously. Thus, the state vector $\mathbf{x}\left(t_{k}\right)$ describing the complete system at time instant $t_{k}$ is

$$
\mathbf{x}\left(t_{k}\right)=\left[\mathbf{x}_{u}\left(t_{k}\right)^{T}, \mathbf{x}_{\mathrm{A}}\left(t_{k}\right)^{T}\right]^{T},
$$

with the tag states $\mathbf{x}_{u}\left(t_{k}\right)$ and the anchor states $\mathbf{x}_{\mathrm{A}}\left(t_{k}\right)$. The tag state $\mathbf{x}_{u}\left(t_{k}\right)$ includes the tag position $\boldsymbol{r}_{u}\left(t_{k}\right)$ and the tag velocity $\boldsymbol{v}_{u}\left(t_{k}\right)$ with

$$
\left.\mathbf{x}_{u}\left(t_{k}\right)=\left[\boldsymbol{r}_{u}\left(t_{k}\right)^{T}, \boldsymbol{v}_{u}\left(t_{k}\right)^{T}\right)\right]^{T},
$$

and the anchor states

$$
\mathbf{x}_{\mathrm{A}}\left(t_{k}\right)=\left[\boldsymbol{r}_{\mathrm{A}, 1}\left(t_{k}\right)^{T}, \ldots, \boldsymbol{r}_{\mathrm{A}, N\left(t_{k}\right)}\left(t_{k}\right)^{T}\right]^{T},
$$

for the $N\left(t_{k}\right)$ anchor positions $\boldsymbol{r}_{\mathrm{A}, i}\left(t_{k}\right)$.

Additionally, as illustrated in Fig. 1, an IMU is used. The IMU provides measurements of the accelerations $\boldsymbol{a}^{b}\left(t_{k}\right)$ and turn rates $\boldsymbol{\omega}_{i b}^{b}\left(t_{k}\right)$ in three dimensions. After calibration, the heading change $\dot{\Psi}\left(t_{k}\right)$ is used as a control input and is therefore directly integrated into the transition model of the pedestrian carrying the tag.

As we consider a dynamic system, the state estimation can be described based on a discrete time formulation by a transition model and a measurement model, with

$$
\begin{aligned}
\mathbf{x}\left(t_{k}\right) & =\mathbf{f}\left(\mathbf{x}\left(t_{k-1}\right), \mathbf{u}\left(t_{k}\right), \mathbf{w}\left(t_{k}\right)\right), \\
\mathbf{z}\left(t_{k}\right) & =\mathbf{h}\left(\mathbf{x}\left(t_{k}\right), \mathbf{n}\left(t_{k}\right)\right) .
\end{aligned}
$$

The transition model in (6) describes the state evolution from time instant $t_{k-1}$ to time instant $t_{k}$ employing a possible nonlinear function $\mathbf{f}(\cdot, \cdot, \cdot)$ using the control input $\mathbf{u}\left(t_{k}\right)$ and process noise $\mathbf{w}\left(t_{k}\right)$. The measurement model (7) relates the state vector to the measurements at time instant $t_{k}$ with a possible nonlinear function $\mathbf{h}(\cdot, \cdot)$ and measurement noise $\mathbf{n}\left(t_{k}\right)$. Fig. 2 shows the considered Dynamic Bayesian Network where we assume a first-order hidden Markov model. 


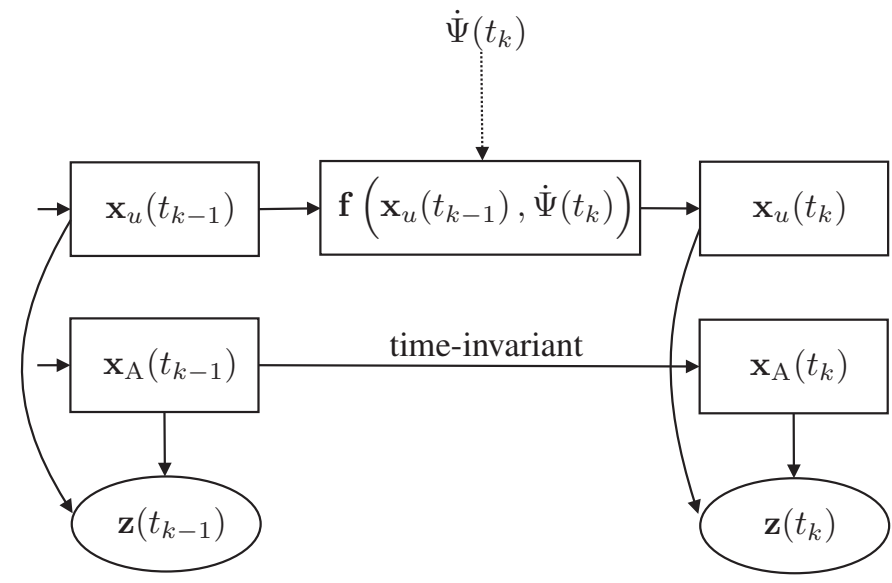

Fig. 2: First-order hidden Markov model representing the dynamic system.

Equations (6) and (7) can also be seen from a Bayesian perspective: based on measurements, we want to recursively estimate the unknown probability density function (PDF) of the state $\mathbf{x}\left(t_{k}\right)$. In a recursive Bayesian formulation, this problem can be described as finding the probability distribution

$$
\begin{aligned}
& \mathrm{p}\left(\mathbf{x}\left(t_{k}\right) \mid \mathbf{z}\left(t_{1: k}\right) \mathbf{u}\left(t_{1: k}\right), \mathbf{x}\left(t_{0}\right)\right) \\
& \quad=\mathbf{p}\left(\mathbf{x}_{u}\left(t_{k}\right), \mathbf{x}_{\mathrm{A}}\left(t_{k}\right) \mid \mathbf{z}\left(t_{1: k}\right) \mathbf{u}\left(t_{1: k}\right), \mathbf{x}_{u}\left(t_{0}\right)\right),
\end{aligned}
$$

with the measurements $\mathbf{z}\left(t_{1: k}\right)=\left[\mathbf{z}\left(t_{1}\right)^{T}, \ldots, \mathbf{z}\left(t_{k}\right)^{T}\right]^{T}$, which are the sets of propagation delays for the time instants $t_{1}, \ldots, t_{k}$, and the control inputs $\mathbf{u}\left(t_{1: k}\right)=$ $\left[\dot{\Psi}\left(t_{1}\right), \ldots, \dot{\Psi}\left(t_{k}\right)\right]^{T}$, respectively. Finding the probability distribution in (8) can be regarded as a SLAM problem [22], [23]. It is the joint posterior density of the anchor states and tag state given the measurements, the control inputs and the initial state of the tag. Fig. 3 visualizes the SLAM process of this work: the tag moves through an environment and takes relative delay measurements between a number of anchors with unknown positions and the tag. In order to use the delay measurements, the localization algorithm estimates the tag and anchor positions simultaneously. The true locations are not known or can not be measured directly.

Based on recursive Bayesian filtering, the posterior distribution $\mathrm{p}\left(\mathbf{x}_{u}\left(t_{k}\right), \mathbf{x}_{\mathrm{A}}\left(t_{k}\right) \mid \mathbf{z}\left(t_{1: k}\right) \mathbf{u}\left(t_{1: k}\right), \mathbf{x}_{u}\left(t_{0}\right)\right)$ can be obtained recursively by a prediction and an update step. In the prediction step, the PDF

$$
\begin{aligned}
& \mathrm{p}\left(\mathbf{x}_{u}\left(t_{k}\right), \mathbf{x}_{\mathrm{A}}\left(t_{k}\right) \mid \mathbf{z}\left(t_{1: k-1}\right), \mathbf{u}\left(t_{1: k}\right), \mathbf{x}_{u}\left(t_{0}\right)\right) \\
& =\int \mathrm{p}\left(\mathbf{x}_{u}\left(t_{k-1}\right), \mathbf{x}_{\mathrm{A}}\left(t_{k-1}\right) \mid \mathbf{z}\left(t_{1: k-1}\right), \mathbf{u}\left(t_{1: k-1}\right), \mathbf{x}_{u}\left(t_{0}\right)\right) \\
& \quad \times \mathrm{p}\left(\mathbf{x}_{u}\left(t_{k}\right) \mid \mathbf{x}_{u}\left(t_{k-1}\right), \mathbf{u}\left(t_{k}\right)\right) \mathrm{d} \mathbf{x}_{u}\left(t_{k-1}\right)
\end{aligned}
$$

is calculated, where we assume a first-order Markov model with the transition prior

$$
\mathrm{p}\left(\mathbf{x}\left(t_{k}\right) \mid \mathbf{x}\left(t_{k-1}\right), \mathbf{u}\left(t_{k}\right)\right) .
$$

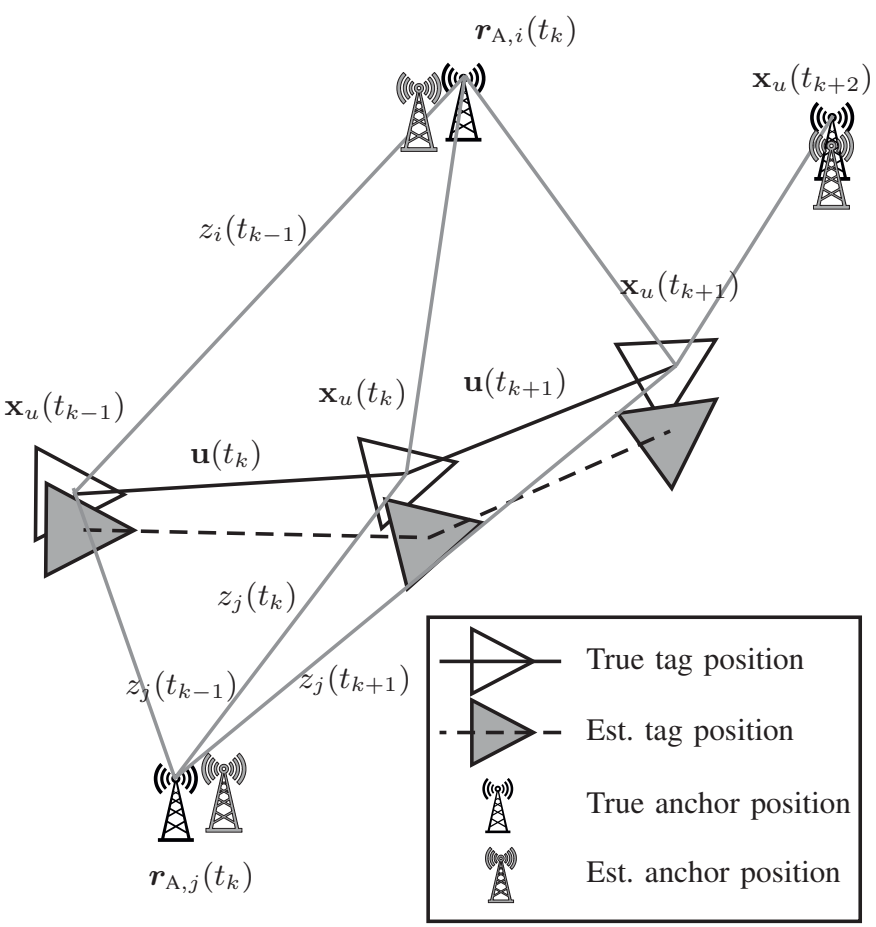

Fig. 3: Overview of the SLAM problem: the moving tag simultaneously estimates its location and the location of the anchors.

The transition prior depends on the state $\mathbf{x}\left(t_{k-1}\right)$ and the applied control input $\mathbf{u}\left(t_{k}\right)$ and is independent of both the observations and the anchor positions. During the update step with

$$
\begin{aligned}
& \mathrm{p}\left(\mathbf{x}_{u}\left(t_{k}\right), \mathbf{x}_{\mathrm{A}}\left(t_{k}\right) \mid \mathbf{z}\left(t_{1: k}\right), \mathbf{u}\left(t_{1: k}\right), \mathbf{x}_{u}\left(t_{0}\right)\right) \\
& =\frac{\mathbf{p}\left(\mathbf{x}_{u}\left(t_{k}\right), \mathbf{x}_{\mathrm{A}}\left(t_{k}\right) \mid \mathbf{z}\left(t_{1: k-1}\right), \mathbf{u}\left(t_{1: k}\right), \mathbf{x}_{u}\left(t_{k-1}\right)\right)}{\mathrm{p}\left(\mathbf{z}\left(t_{k}\right) \mid \mathbf{z}\left(t_{1: k-1}\right), \mathbf{u}\left(t_{1: k}\right)\right)} \\
& \quad \times \mathbf{p}\left(\mathbf{z}\left(t_{k}\right) \mid \mathbf{x}_{u}\left(t_{k}\right), \mathbf{x}_{\mathrm{A}}\left(t_{k}\right)\right),
\end{aligned}
$$

the measurement $\mathbf{z}\left(t_{k}\right)$ is used to correct the prediction based on the measurement likelihood

$$
\mathrm{p}\left(\mathbf{z}\left(t_{k}\right) \mid \mathbf{x}_{u}\left(t_{k}\right), \mathbf{x}_{\mathrm{A}}\left(t_{k}\right)\right) .
$$

Equations (9) and (11) provide a recursive procedure for calculating the joint posterior $\mathrm{p}\left(\mathbf{x}_{u}\left(t_{k}\right), \mathbf{x}_{\mathrm{A}}\left(t_{k}\right) \mid \mathbf{z}\left(t_{1: k}\right) \mathbf{u}\left(t_{1: k}\right), \mathbf{x}_{u}\left(t_{0}\right)\right)$ for the tag state $\mathbf{x}_{u}\left(t_{k}\right)$ and anchors $\mathbf{x}_{\mathrm{A}}\left(t_{k}\right)$ at time instant $t_{k}$ based on all measurements $\mathbf{z}\left(t_{1: k}\right)$ and all control inputs $\mathbf{u}\left(t_{1: k}\right)$. In the following we describe the transition and the measurement model.

\section{A. Transition Model}

The transition prior $\mathrm{p}\left(\mathbf{x}\left(t_{k}\right) \mid \mathbf{x}\left(t_{k-1}\right), \mathbf{u}\left(t_{k}\right)\right)$ of the receiver state vector $\mathbf{x}_{u}\left(t_{k}\right)$ and the anchor state vectors $\mathbf{x}_{\mathrm{A}, i}\left(t_{k}\right)$ associated to the anchors $i=1, \ldots, N\left(t_{k}\right)$, is

$$
\mathrm{p}\left(\mathbf{x}\left(t_{k}\right) \mid \mathbf{x}\left(t_{k-1}\right), \mathbf{u}\left(t_{k}\right)\right)=\mathrm{p}\left(\mathbf{x}_{u}\left(t_{k}\right) \mid \mathbf{x}_{u}\left(t_{k-1}\right), \mathbf{u}\left(t_{k}\right)\right)
$$




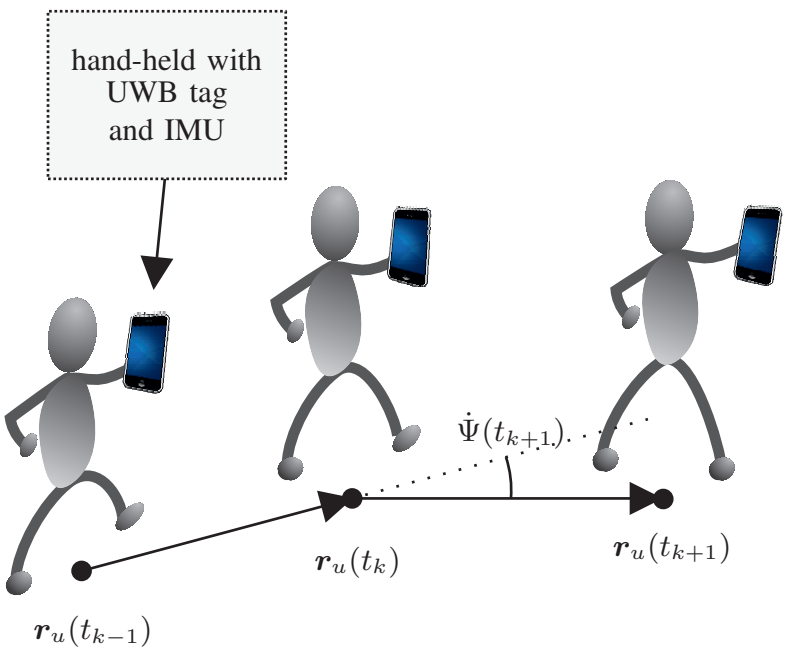

Fig. 4: Illustration of the prediction model for the pedestrian.

$$
\times \prod_{i=1}^{N\left(t_{k}\right)} \mathrm{p}\left(\mathbf{x}_{\mathrm{A}, i}\left(t_{k}\right) \mid \mathbf{x}_{\mathrm{A}, i}\left(t_{k-1}\right)\right) \text {. }
$$

The anchor states $\mathbf{x}_{\mathrm{A}, i}\left(t_{k}\right)$ are time-invariant, hence, we obtain for the transition prior $\mathrm{p}\left(\mathrm{x}_{\mathrm{A}, i}\left(t_{k}\right) \mid \mathrm{x}_{\mathrm{A}, i}\left(t_{k-1}\right)\right)$ of the $i$-th anchor

$$
\begin{aligned}
\mathrm{p}\left(\mathbf{x}_{\mathrm{A}, i}\left(t_{k}\right) \mid \mathbf{x}_{\mathrm{A}, i}\left(t_{k-1}\right)\right) \\
=\delta\left(\mathbf{x}_{\mathrm{A}, i}\left(t_{k}\right)-\mathbf{x}_{\mathrm{A}, i}\left(t_{k-1}\right)\right) .
\end{aligned}
$$

To describe the transition prior $\mathrm{p}\left(\mathbf{x}_{u}\left(t_{k}\right) \mid \mathbf{x}_{u}\left(t_{k-1}\right), \mathbf{u}\left(t_{k}\right)\right)$ of the tag state vector we consider a pedestrian holding a hand-held device as shown in Fig. 4. The hand-held device is equipped with the UWB tag and an IMU. For the movement model, we follow a strapdown approach [32]. IMUs include in general accelerometers measuring accelerations $\boldsymbol{a}^{b}\left(t_{k}\right)$ and gyroscopes measuring turn rates $\boldsymbol{\omega}_{i b}^{b}\left(t_{k}\right)$, as indicated in Fig. 1. These measurements are provided with respect to the sensor alignment [32], i.e., the body frame. In order to obtain the measurements in a two-dimensional Cartesian coordinate system as shown in Fig. 4, a transformation between the coordinate systems is necessary, see e.g. [33]. In our considered measurement scenario, the position of the IMU is assumed as constant with respect to the pedestrian. Therefore, we are able to calculate the coordinate transformation matrices during the calibration phase when the pedestrian is standing at the beginning of the measurements. For practical systems, where the sensor is decoupled, the sensor orientation has to be estimated continuously by applying strapdown navigation together with in-field calibration [34].

As indicated in Fig. 4, we follow a two-dimensional positioning approach in the Cartesian coordinate system with the receiver position

$$
\boldsymbol{r}_{u}\left(t_{k}\right)=\left[r_{u, x}\left(t_{k}\right), r_{u, y}\left(t_{k}\right)\right]^{T},
$$

where $r_{u, x}\left(t_{k}\right)$ and $r_{u, y}\left(t_{k}\right)$ denote the $\mathrm{x}-\mathrm{y}$ positions. The receiver velocity state is

$$
\boldsymbol{v}_{u}\left(t_{k}\right)=\mathbf{R}\left(\tilde{\Psi}\left(t_{k}\right)\right) \cdot \boldsymbol{v}_{u}\left(t_{k-1}\right)+\mathbf{n}_{u}\left(t_{k}\right),
$$

with the rotation matrix

$$
\mathbf{R}\left(\tilde{\Psi}\left(t_{k}\right)\right)=\left(\begin{array}{cc}
\cos \left(\tilde{\Psi}\left(t_{k}\right)\right) & -\sin \left(\tilde{\Psi}\left(t_{k}\right)\right) \\
\sin \left(\tilde{\Psi}\left(t_{k}\right)\right) & \cos \left(\tilde{\Psi}\left(t_{k}\right)\right)
\end{array}\right),
$$

and

$$
\tilde{\Psi}\left(t_{k}\right)=\dot{\Psi}\left(t_{k}\right)+w_{\Psi}\left(t_{k}\right),
$$

which includes the heading rate $\dot{\Psi}\left(t_{k}\right)$ measurements from the IMU after calibration, and the heading noise $w_{\Psi}\left(t_{k}\right)$.

\section{B. Measurement Model}

For the measurement likelihood $\mathrm{p}\left(\mathbf{z}\left(t_{k}\right) \mid \mathbf{x}\left(t_{k}\right)\right)$, we assume that the elements of $\mathbf{z}\left(t_{k}\right)$ are independent Gaussian distributed conditioned on the current state $\mathbf{x}\left(t_{k}\right)$,

$$
\mathrm{p}\left(\mathbf{z}\left(t_{k}\right) \mid \mathbf{x}\left(t_{k}\right)\right)=\prod_{i=1}^{N\left(t_{k}\right)} \frac{1}{\sqrt{2 \pi} \sigma_{d, i}\left(t_{k}\right)} e^{-\frac{\left(\hat{d}_{i}\left(t_{k}\right)-\tilde{d}_{i}\left(t_{k}\right)\right)^{2}}{2 \sigma_{d, i}^{2}\left(t_{k}\right)}},
$$

with the distance estimate $\hat{d}_{i}\left(t_{k}\right)$, the propagation length $\tilde{d}_{i}\left(t_{k}\right)$ and the corresponding variance $\sigma_{d, i}^{2}\left(t_{k}\right)$ for the $i$-th anchor. The propagation length $\tilde{d}_{i}\left(t_{k}\right)$ is

$$
\tilde{d}_{i}\left(t_{k}\right)=\left\|\boldsymbol{r}_{u}\left(t_{k}\right)-\boldsymbol{r}_{\mathrm{A}, i}\left(t_{k}\right)\right\|+d_{b}\left(t_{k}\right)
$$

which is similar to $d_{i}\left(t_{k}\right)$ of (1) with an additional measurement error $d_{b}\left(t_{k}\right)$ to include NLoS, multipath and general estimation errors.

\section{Particle Filter Implementation}

In this section, we summarize the particle filter (PF) implementation of the algorithm derived in Section II. PFs provide numerical approximations to the nonlinear Bayesian filtering problem. They approximate the posterior density of the state vector $\mathbf{x}\left(t_{k}\right)$ at time step $t_{k}$ by $N_{p}$ particles with the particle states $\mathbf{x}^{(j)}\left(t_{k}\right)$ and associated weights $w^{(j)}\left(t_{k}\right)$ with $j=1, \ldots, N_{p}$ [35]-[37]. The implementation is similar to the Channel-SLAM algorithm presented in [38]. We implemented the algorithm based on Rao-Blackwellization [39]. The posterior density $\mathrm{p}\left(\mathbf{x}_{u}\left(t_{k}\right), \mathbf{x}_{\mathrm{A}}\left(t_{k}\right) \mid \mathbf{z}\left(t_{1: k}\right) \mathbf{u}\left(t_{1: k}\right), \mathbf{x}_{u}\left(t_{0}\right)\right)$ of (8) can be written as

$$
\begin{aligned}
\mathrm{p}\left(\mathbf{x}_{u}\left(t_{k}\right), \mathbf{x}_{\mathrm{A}}\left(t_{k}\right) \mid \mathbf{z}\left(t_{1: k}\right) \mathbf{u}\left(t_{1: k}\right), \mathbf{x}_{u}\left(t_{0}\right)\right) \\
=\mathrm{p}\left(\mathbf{x}_{u}\left(t_{k}\right) \mid \mathbf{z}\left(t_{0: k}\right), \mathbf{u}\left(t_{1: k}\right), \mathbf{x}_{u}\left(t_{0}\right)\right) \\
\mathrm{p}\left(\mathbf{x}_{\mathrm{A}}\left(t_{k}\right) \mid \mathbf{x}_{u}\left(t_{k}\right), \mathbf{z}\left(t_{0: k}\right)\right) \\
=\mathrm{p}\left(\mathbf{x}_{u}\left(t_{k}\right) \mid \mathbf{z}\left(t_{0: k}\right), \mathbf{u}\left(t_{1: k}\right), \mathbf{x}_{u}\left(t_{0}\right)\right) \\
\quad \times \prod_{i=1}^{N\left(t_{k}\right)} \mathrm{p}\left(\mathbf{x}_{\mathrm{A}, i}\left(t_{k}\right) \mid \mathbf{x}_{u}\left(t_{k}\right), \mathbf{z}_{i}\left(t_{0: k}\right)\right) .
\end{aligned}
$$

Hence, the RBPF contains additionally to the samples $\mathbf{x}_{u}^{(j)}\left(t_{k}\right)$ from $\mathrm{p}\left(\mathbf{x}_{u}\left(t_{k}\right) \mid \mathbf{z}\left(t_{0: k}\right), \mathbf{u}\left(t_{1: k}\right), \mathbf{x}_{u}\left(t_{0}\right)\right)$ a representation of anchor states $\mathrm{p}\left(\mathbf{x}_{\mathrm{A}}\left(t_{k}\right) \mid \mathbf{x}_{u}^{(j)}\left(t_{k}\right), \mathbf{z}\left(t_{0: k}\right)\right)$ for each particle $j$. We use PFs to estimate the subspaces representing the anchor 
states inside a PF. The reason to use a PF instead of a low complexity EKF is the non-linearity of the measurements in (1). As shown in Fig. 5, the algorithm is based on a superordinate particle filter (superPF) and subordinate particle filters (subPFs): Each particle $j=1, \ldots, N_{p}$ of the superPF with the state vector $\mathbf{x}_{u}^{(j)}\left(t_{k}\right)=\left[\boldsymbol{r}_{u}^{(j)}\left(t_{k}\right)^{T}, \boldsymbol{v}_{u}^{(j)}\left(t_{k}\right)^{T}\right]^{T}$ holds $N\left(t_{k}\right)$ subPFs. Each subPF is represented by the particles $\mathbf{x}_{\mathrm{A}, i}^{(j, a)}\left(t_{k}\right)$ with $a=1, \ldots, N_{P, j, i}\left(t_{k}\right)$ where $N_{P, j, i}\left(t_{k}\right)$ stands for the number of particles in the $i$-th subPF with $i=1, \ldots, N\left(t_{k}\right)$, estimating $\mathbf{x}_{\mathrm{A}, i}^{(j)}\left(t_{k}\right)$.

Consequently, similar to [38], the posterior density $\mathrm{p}\left(\mathbf{x}_{u}\left(t_{k}\right), \mathbf{x}_{\mathrm{A}}\left(t_{k}\right) \mid \mathbf{z}\left(t_{1: k}\right) \mathbf{u}\left(t_{1: k}\right), \mathbf{x}_{u}\left(t_{0}\right)\right)$ can be approximated by importance samples, see [36], as

$$
\begin{gathered}
\mathrm{p}\left(\mathbf{x}_{u}\left(t_{k}\right), \mathbf{x}_{\mathrm{A}}\left(t_{k}\right) \mid \mathbf{z}\left(t_{1: k}\right) \mathbf{u}\left(t_{1: k}\right), \mathbf{x}_{u}\left(t_{0}\right)\right) \\
\approx \sum_{j=1}^{N_{s}} w^{(j)}\left(t_{k}\right) \delta\left(\mathbf{x}_{u}\left(t_{k}\right)-\mathbf{x}_{u}^{(j)}\left(t_{k}\right)\right),
\end{gathered}
$$

where $w^{(j)}\left(t_{k}\right)$ defines the weight for the $j$-th particle at time instant $t_{k}$ with

$$
\begin{aligned}
w^{(j)}\left(t_{k}\right) & \propto \mathrm{p}\left(\mathbf{z}\left(t_{k}\right) \mid \mathbf{x}_{u}^{(j)}\left(t_{k}\right), \mathbf{z}\left(t_{k-1}\right)\right) \\
& \propto \prod_{i=1}^{N(t)} \sum_{a=1}^{N_{P, i, j}\left(t_{k}\right)} w_{i}^{(j, a)}\left(t_{k}\right)
\end{aligned}
$$

and the weight $w_{i}^{(j, a)}\left(t_{k}\right)$ of the subPFs at time instant $t_{k}$ with

$$
w_{i}^{(j, a)}\left(t_{k}\right) \triangleq \mathrm{p}\left(\hat{d}_{i}\left(t_{k}\right) \mid \mathbf{x}_{u}^{(j)}\left(t_{k}\right), \mathbf{x}_{\mathrm{A}, i}^{(j, a)}\left(t_{k}\right)\right) .
$$

Resampling is performed at each time instant to prevent degeneration, hence, (24) and (25) do not depend on the weights $w^{(j)}\left(t_{k-1}\right)$ and $w_{i}^{(j, a)}\left(t_{k-1}\right)$, respectively.

\section{Measurement Equipment and Scenario}

\section{A. Measurement Equipment}

The pedestrian is equipped with a hand-held device including an UWB tag, a Xsense IMU (MTI-G-700) and a laptop which stores the IMU and UWB measurement data. We use the so called Loco Positioning system produced by Bitcraze AB, see https://www.bitcraze.io/ for our measurements. The Loco Positioning system is based on the Decawave DWM1000 chip and has an accuracy in the $10 \mathrm{~cm}$ range. For the measurements, the UWB system is configured to a bandwidth of $500 \mathrm{MHz}$ and a carrier frequency of $3.5 \mathrm{GHz}$. Fig. 6 shows the Loco Positioning Node which can act either as an anchor or as a tag. As an anchor, it is part of the reference base for the system. In tag mode, it can be connected to a computer for recording the distance estimates.

The UWB system uses a two way ranging method as depicted in Fig. 7 which allows the tag to measure its distance to an anchor $i$. The protocol contains four messages, two are sent by the tag, and two by the anchor. Afterwards, the
TABLE I: Description of the track-markers

\begin{tabular}{cc}
\hline Marker & Time instants \\
\hline $\mathbf{M}_{1}$ & $t=0 \mathrm{~s}$, \\
\hline $\mathbf{M}_{2}$ & $t=13.8 \mathrm{~s}, t=61.7 \mathrm{~s}$ \\
\hline $\mathbf{M}_{3}$ & $t=17.2 \mathrm{~s}, t=64.8 \mathrm{~s}$ \\
\hline $\mathbf{M}_{4}$ & $t=29 \mathrm{~s}, t=75.1 \mathrm{~s}$ \\
\hline $\mathrm{M}_{5}$ & $t=41 \mathrm{~s} t=43.6 \mathrm{~s}$ \\
\hline $\mathrm{M}_{6}$ & $t=79.1 \mathrm{~s}$ \\
\hline $\mathrm{M}_{7}$ & $t=87.2 \mathrm{~s}$ \\
\hline
\end{tabular}

propagation delay is estimated by

$$
\hat{d}_{i}\left(t_{k}\right)=c \cdot \frac{\Delta_{\tau, 1, i}\left(t_{k}\right) \cdot \Delta_{\tau, 4, i}\left(t_{k}\right)-\Delta_{\tau, 2, i}\left(t_{k}\right) \cdot \Delta_{\tau, 3, i}\left(t_{k}\right)}{\Delta_{\tau, 1, i}\left(t_{k}\right) \cdot \Delta_{\tau, 4, i}\left(t_{k}\right)+\Delta_{\tau, 2, i}\left(t_{k}\right) \cdot \Delta_{\tau, 3, i}\left(t_{k}\right)},
$$

based on the transmitting and receiving time stamps illustrated in Fig. 7.

\section{B. Measurement Scenario}

Fig. 8 shows the indoor measurement scenario in top view with the five anchor positions indicated by $\mathrm{A}_{i}$ for $i=1, \ldots, 5$ and the pedestrian track in blue. The measurements were conducted in the kitchen area of an office building. The pedestrian is moving on the indicated blue track starting at $\mathrm{M}_{1}$ and ending at $\mathrm{M}_{7}$ for $87 \mathrm{~s}$ in the order: $\mathrm{M}_{1} \Rightarrow \mathrm{M}_{2} \Rightarrow$ $\mathrm{M}_{3} \Rightarrow \mathrm{M}_{4} \Rightarrow \mathrm{M}_{5} \Rightarrow \mathrm{M}_{2} \Rightarrow \mathrm{M}_{3} \Rightarrow \mathrm{M}_{4} \Rightarrow \mathrm{M}_{6} \Rightarrow \mathrm{M}_{7}$. During the pedestrian movement, the line-of-sight (LoS) path between the anchors and the tag is most of the time present, however sometimes shaded by the body of the pedestrian or by furniture. Fig. 9 shows the estimated propagation distances $\hat{d}_{i}\left(t_{k}\right)$ in meter versus the pedestrian moving time in seconds. Additionally, the figure shows by the black lines the corresponding calculated propagation distances, which match to the UWB measurements.

\section{Evaluations Based on Measurements}

The evaluations are performed using $N_{s}=2000$ particles in the superPF, whereas the number of particles for the subPFs for each node is different depending on the estimated delay. The developed algorithm obtains the measurements $\mathbf{z}\left(t_{k}\right)$ and the heading rate $\dot{\Psi}\left(t_{k}\right)$ every $T=t_{k}-t_{k-1}=0.1 \mathrm{~s}$. The error in the heading noise of (19) is modeled by a Gaussian density $w_{\Psi}\left(t_{k}\right) \sim \mathcal{N}\left(0, \sigma_{\Phi}^{2}\left(t_{k}\right)\right)$ with standard deviation $\sigma_{\Phi}\left(t_{k}\right)=$ $1^{\circ}$. For the initialization, we use prior information $\mathrm{p}\left(\mathbf{x}_{u}\left(t_{0}\right)\right)$ including the starting position and velocity. Being a relative positioning system, the algorithm requires prior knowledge of the initial receiver position and moving direction only to define a local coordinate system. Hence, an unknown starting position and direction or larger initial uncertainties may result in a biased and rotated coordinate system in the estimation.

To evaluate the positioning performance of the proposed algorithm, we compare it to a positioning algorithm with perfect knowledge of all anchor positions. In the following we refer to this algorithm as UWB Pos., which can be seen as 


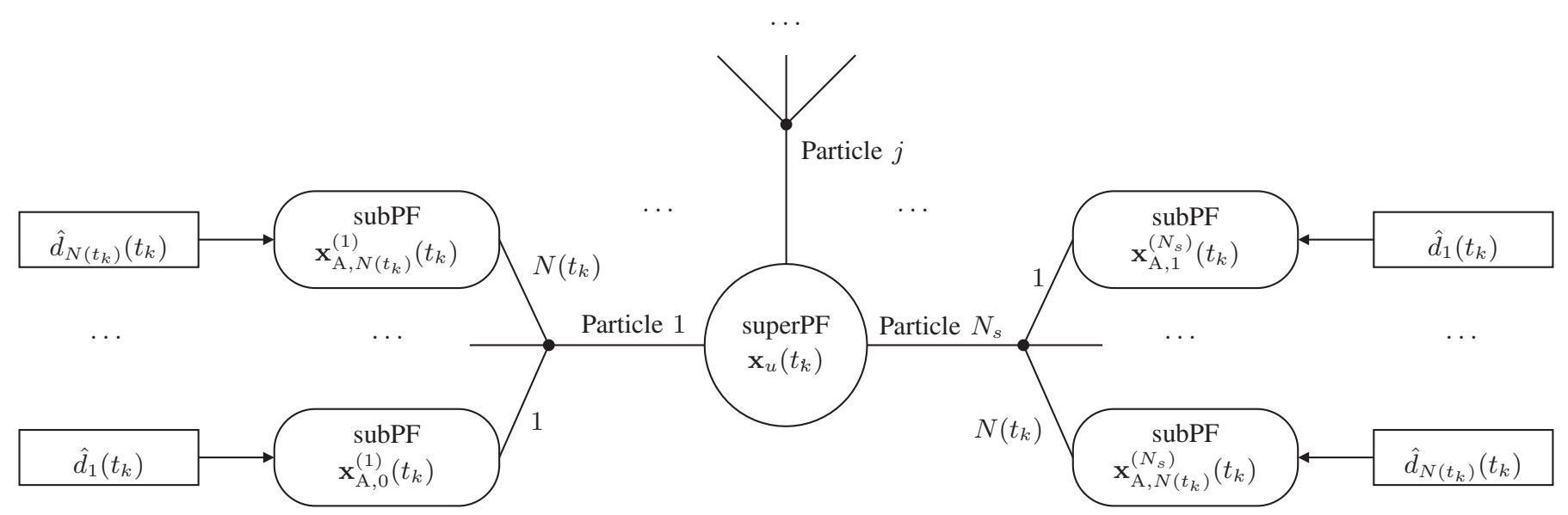

Fig. 5: The algorithm is based on a superordinate particle filter (superPF) and subordinate particle filters (subPFs). Each particle $j=1 \ldots N_{s}$ of the superPF holds $N\left(t_{k}\right)$ subPFs.

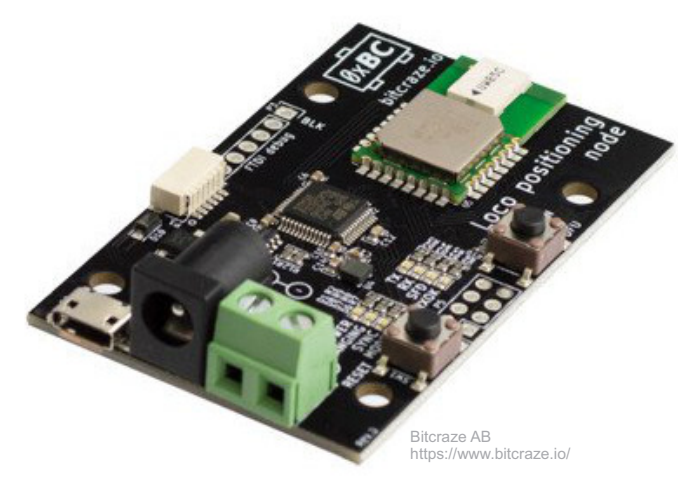

Fig. 6: The Loco Positioning Node is a multi functional node in a Loco Positioning system which can act either as an anchor or tag.

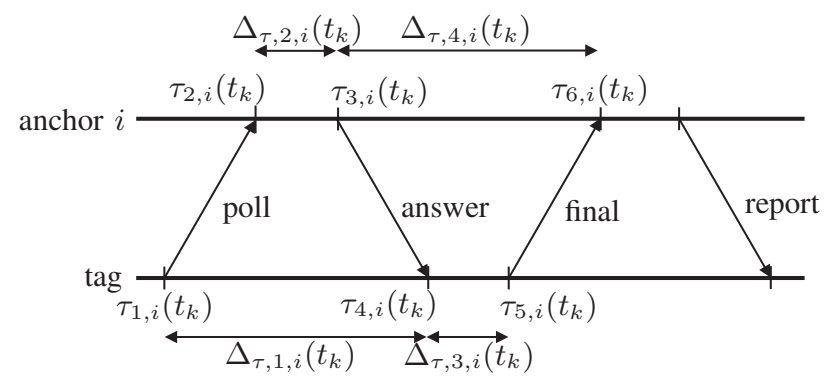

Fig. 7: The two way ranging protocol allows a tag to measure its distance to an anchor.

a lower bound for the proposed algorithm referred to as $U W B$ SLAM. We assume the starting position and direction of the pedestrian to be known. Fig. 10 shows the root mean square errors (RMSEs) $\operatorname{RMSE}_{u}\left(t_{k}\right)=\sqrt{\mathbf{E}\left\{\left\|\boldsymbol{r}_{u}\left(t_{k}\right)-\hat{\boldsymbol{r}}_{u}\left(t_{k}\right)\right\|^{2}\right\}}$ of the estimated pedestrian position versus the pedestrian moving time for UWB SLAM in blue and UWB Pos. in green. Because the PF includes randomness, the position estimates differ for each evaluation due to a finite number of particles even if

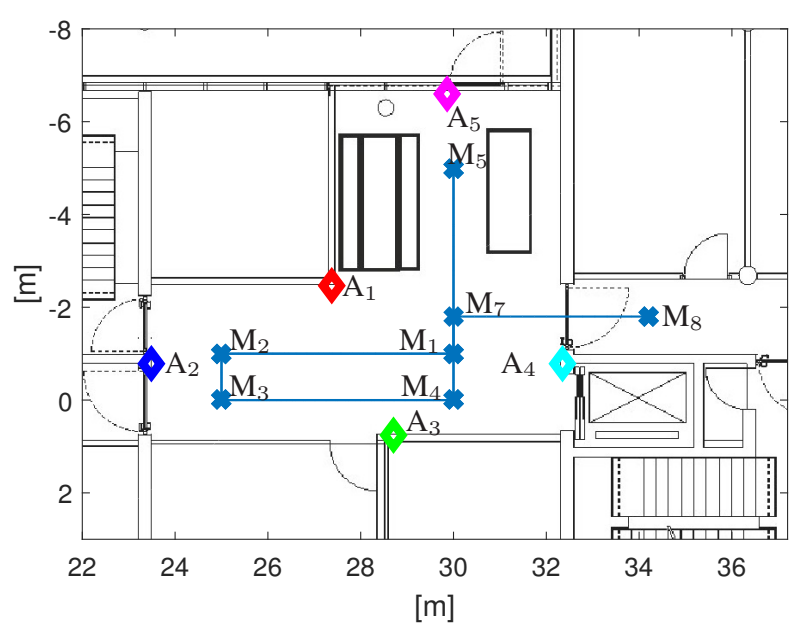

Fig. 8: Measurement scenario with five fixed anchors $\left(A_{1}, A_{2}\right.$, $\mathrm{A}_{3}, \mathrm{~A}_{4}$ and $\mathrm{A}_{5}$ ) and a moving tag (pedestrian). The pedestrian moves on the blue track for $85 \mathrm{~s}$ starting at $\mathrm{M}_{1}$ and ending at $\mathrm{M}_{7}$ in the order: $\mathrm{M}_{1} \Rightarrow \mathrm{M}_{2} \Rightarrow \mathrm{M}_{3} \Rightarrow \mathrm{M}_{4} \Rightarrow \mathrm{M}_{5} \Rightarrow \mathrm{M}_{2} \Rightarrow$ $\mathrm{M}_{3} \Rightarrow \mathrm{M}_{4} \Rightarrow \mathrm{M}_{6} \Rightarrow \mathrm{M}_{7}$, see also Table I.

the same measurement data are used. Therefore, we perform 200 independent evaluations based on the same measurement data. Because of the initialization of the receiver position using prior knowledge, both algorithms perform similarly at the beginning of the track, where the position error is rather low. Afterwards, the $\operatorname{RMSE}_{u}\left(t_{k}\right)$ for both algorithms varies between $0.1 \mathrm{~m}$ and $1 \mathrm{~m}$. UWB Pos. can be interpreted as a lower bound and estimates the receiver position with the lowest RMSE. However, at some time steps UWB Pos. has a slightly higher RMSE than UWB SLAM which might be due to the small inaccuracies in the calculation of the VT positions. Fig. 11 shows the cumulative distribution functions (CDFs) of the error of the estimated receiver position for the mentioned algorithms. In order to reduce the effect of the initialization, the CDFs do not consider the first $4 \mathrm{~s}$ of the pedestrian movement. 


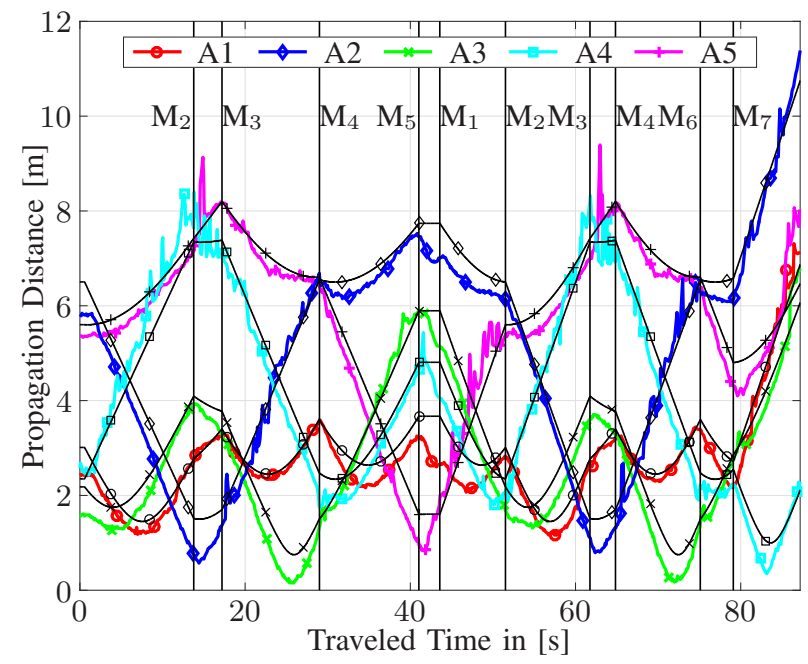

Fig. 9: Recorded delays versus the pedestrian moving time in seconds.

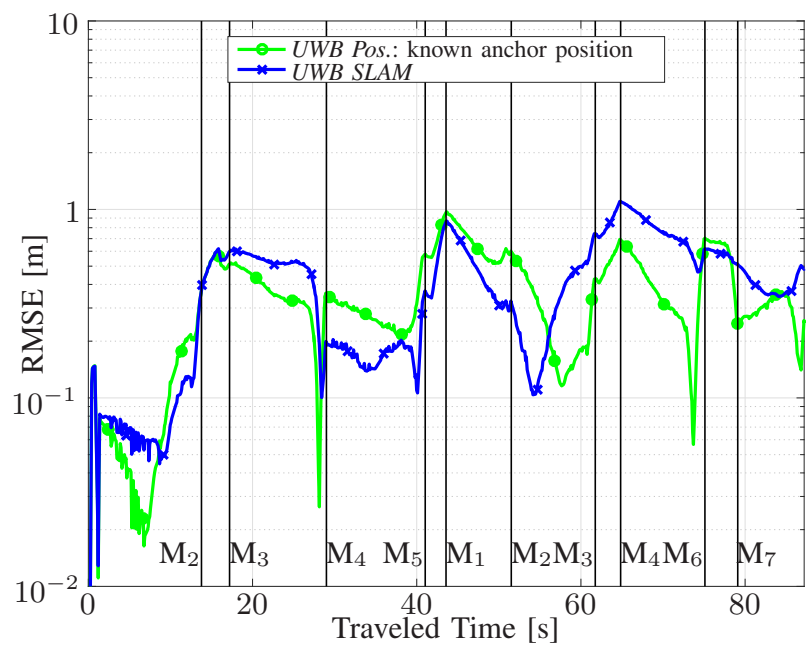

Fig. 10: $\operatorname{RMSE}_{u}\left(t_{k}\right)$ versus the pedestrian moving time in seconds for the described SLAM algorithm and a positioning algorithm with knowledge of the anchor positions.

Fig. 12 shows the RMSE of the estimated positions of the anchors $\mathrm{A}_{i}, \operatorname{RMSE}_{\mathrm{A}_{i}}\left(t_{k}\right)=\sqrt{\mathbf{E}\left\{\left\|\boldsymbol{r}_{\mathrm{A}, i}\left(t_{k}\right)-\hat{\boldsymbol{r}}_{\mathrm{A}, i}\left(t_{k}\right)\right\|^{2}\right\}}$, for $i=1, \ldots, 5$, versus the pedestrian moving time. At the starting position, the algorithm has no prior knowledge of the anchor positions. The RBPF is initialized based on the first delay estimates. Hence, the possible position of anchor $\mathrm{A}_{i}$ lies within a circle around $\boldsymbol{r}_{u}\left(t_{0}\right)$ with radius $\hat{d}_{i}\left(t_{0}\right) .{ }^{1}$ During the pedestrian movement the positions of the anchors converge and the RMSE decreases. At the end of the track we can estimate all anchors with a RMSE lower than $0.5 \mathrm{~m}$. Especially, the RMSE of $\mathrm{A}_{1}$ is below $0.05 \mathrm{~m}$.

\footnotetext{
${ }^{1}$ Please note, we are considering positioning in a two-dimensional coordinate system, where the anchors and tag are on the same level. If the anchors are placed e.g. on the ceiling, the possible position of the anchor $\mathrm{A}_{i}$ lies within a sphere around $\boldsymbol{r}_{u}\left(t_{0}\right)$ with radius $\hat{d}_{i}\left(t_{0}\right)$.
}



Fig. 11: CDF of the receiver position error for UWB Pos. and $U W B S L A M$. In order to reduce the effect of the initialization around the starting position, the evaluations do not consider the first $5 \mathrm{~s}$ of the pedestrian movement.

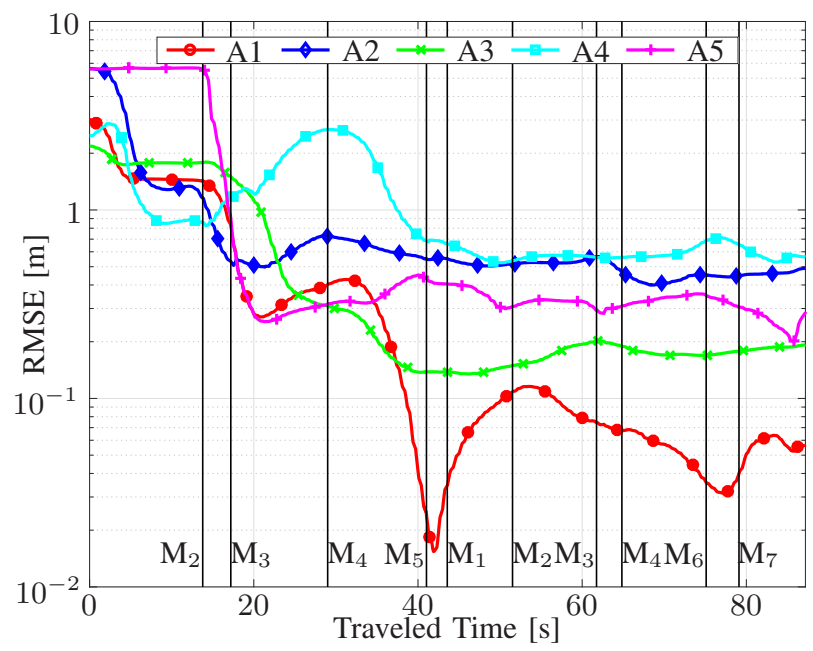

Fig. 12: $\operatorname{RMSE}_{\mathrm{A}_{i}}\left(t_{k}\right)$ versus the pedestrian moving time in seconds for the anchors $A_{1}, A_{2}, A_{3}, A_{4}$ and $A_{5}$

\section{Conclusions}

In this paper, we presented a SLAM algorithm for localization of a pedestrian using a UWB system with unknown anchor positions. The tag and anchor positions are estimated simultaneously based on range measurements between the anchors and the tag. Our evaluations show that an accurate positioning performance can be achieved even if the anchor positions are unknown. The pedestrian RMSE was almost always below one meter, and in $90 \%$ of the time below $0.77 \mathrm{~m}$. In addition, the locations of the five anchors could be estimated with final RMSEs between 0.05 and $0.5 \mathrm{~m}$.

\section{ACKNOWLEDGEMENT}

This work has been performed in the framework of the DLR project Navigation 4.0 and the European Unions Horizon 
2020 research and innovation programme under grant agreement No. 636537 HIGHTS (High precision positioning for Cooperative-ITS applications).

\section{REFERENCES}

[1] G. Seco-Granados, J. López-Salcedo, D. Jiménez-Baños, and G. LópezRisueño, "Challenges in Indoor Global Navigation Satellite Systems," IEEE Signal Process. Mag., vol. 29, no. 2, pp. 108-131, Mar. 2012.

[2] H. Liu, H. Darabi, P. Banerjee, and J. Liu, "Survey of Wireless Indoor Positioning Techniques and Systems," IEEE Trans. Syst., Man, Cybern. C, vol. 37, no. 6, pp. 1067-1080, Nov. 2007.

[3] D. Dardari, P. Closas, and P. M. Djuri?, "Indoor Tracking: Theory, Methods, and Technologies," IEEE Trans. Veh. Technol., vol. 64, no. 4, pp. 1263-1278, Apr. 2015.

[4] "Performance-based evaluation of rfid-based indoor location sensing solutions for the built environment," Adv. Eng. Inf., vol. 25, no. 3, pp. 535 - 546, Aug. 2011.

[5] A. H. Sayed, A. Tarighat, and N. Khajehnouri, "Network-Based Wireless Location: Challenges Faced in Developing Techniques for Accurate Wireless Location Information," IEEE Signal Process. Mag., vol. 22, pp. 24-40, Jul. 2005.

[6] Y. Zhao, "Standardization of Mobile Phone Positioning for 3G Systems," IEEE Commun. Mag., vol. 40, no. 7, pp. 108-116, Jul. 2002.

[7] K. Kaemarungsi and P. Krishnamurthy, "Properties of Indoor Received Signal Strength for WLAN Location Fingerprinting," Aug. 2004, pp. $14-23$.

[8] M. Win and R. Scholtz, "Characterization of Ultra-Wide Bandwidth Wireless Indoor Channels: a Communication-Theoretic View," IEEE J. Sel. Areas Commun., vol. 20, no. 9, pp. 1613-1627, Dec. 2002.

[9] A. Molisch, D. Cassioli, C.-C. Chong, S. Emami, A. Fort, B. Kannan, J. Karedal, J. Kunisch, H. Schantz, K. Siwiak, and M. Win, "A Comprehensive Standardized Model for Ultrawideband Propagation Channels," IEEE Trans. Antennas Propag., vol. 54, no. 11, pp. 3151-3166, Nov. 2006.

[10] C. Steiner and A. Wittneben, "Low Complexity Location Fingerprinting With Generalized UWB Energy Detection Receivers," IEEE Trans. Signal Process., vol. 58, no. 3, pp. 1756-1767, Mar. 2010.

[11] I. Oppermann, M. Hämäläinen, and J. Iinatti, UWB: Theory and Applications. John Wiley \& Sons, 2005.

[12] Y. Luo, O. Hoeber, and Y. Chen, "Enhancing Wi-Fi fingerprinting for indoor positioning using human-centric collaborative feedback," Humancentric Computing and Information Sciences, vol. 3, no. 1, p. 2, 2013.

[13] P. Bahl and V. N. Padmanabhan, "RADAR: an In-Building RF-Based User Location and Tracking System," in Proc. IEEE INFOCOM 2000. Conf. on Comp. Commun., vol. 2, 2000, pp. 775-784 vol.2.

[14] G. Shi and Y. Ming, "Survey of Indoor Positioning Systems Based on Ultra-wideband (UWB) Technology," in Wireless Communications, Networking and Applications. Springer, 2016, pp. 1269-1278.

[15] H. Liu, H. Darabi, P. Banerjee, and J. Liu, "Survey of Wireless Indoor Positioning Techniques and Systems," IEEE Transactions on Systems, Man, and Cybernetics, Part C (Applications and Reviews), vol. 37, no. 6, pp. 1067-1080, 2007.

[16] Z. Sahinoglu, S. Gezici, and I. Guvenc, "Ultra-Wideband Positioning Systems," Cambridge, New York, 2008

[17] M. Kok, J. D. Hol, and T. B. Schön, "Indoor Positioning using Ultrawideband and Inertial Measurements," IEEE Trans. Veh. Technol., vol. 64, no. 4, pp. 1293-1303, 2015.

[18] S. Lee, B. Kim, H. Kim, R. Ha, and H. Cha, "Inertial sensor-based indoor pedestrian localization with minimum 802.15 . 4a configuration," IEEE Trans. Ind. Informat., vol. 7, no. 3, pp. 455-466, 2011.

[19] R. Smith and P. Cheeseman, "On the Representation and Estimation of Spatial Uncertainty," vol. 5, no. 4, pp. 56-68, 1986.

[20] R. Smith, M. Self, and P. Cheeseman, "Estimating Uncertain Spatial Relationships in Robotics," in Proc. Second Annual Conf. on Uncertainty in Artificial Intelligence, University of Pennsylvania, Philadelphia, USA, 1986.

[21] J. Leonard and H. Durrant-whyte, "Simultaneous Map Building and Localization For an Autonomous Mobile Robot," in Proc. IEEE/RSJ Int. Workshop on Intelligent Robots and Systems, Osaka, Japan, Nov. 1991.

[22] H. Durrant-Whyte and T. Bailey, "Simultaneous Localization and Mapping: Part I," IEEE Robot. Autom. Mag., vol. 13, no. 2, pp. 99-110, Jun. 2006.
[23] T. Bailey and H. Durrant-Whyte, "Simultaneous Localization and Mapping (SLAM): Part II," IEEE Robot. Autom. Mag., vol. 13, no. 3, pp. 108-117, 2006

[24] M. G. Dissanayake, P. Newman, S. Clark, H. F. Durrant-Whyte, and M. Csorba, "A Solution to the Simultaneous Localization and Map Building (SLAM) Problem," IEEE Trans. Robot. Autom., vol. 17, no. 3, pp. 229-241, 2001

[25] S. Thrun and M. Montemerlo, "The Graph SLAM Algorithm with Applications to Large-Scale Mapping of Urban Structures," The International Journal of Robotics Research, vol. 25, no. 5-6, pp. 403-429, 2006.

[26] M. Montemerlo, S. Thrun, D. Koller, B. Wegbreit et al., "FastSLAM: A factored solution to the simultaneous localization and mapping problem," in Proc. AAAI Nat. Conf. on Artificial Intelligence, Edmonton, Canada, 2002, pp. 593-598.

[27] A. J. Davison, I. D. Reid, N. D. Molton, and O. Stasse, "MonoSLAM Real-Time Single Camera SLAM," IEEE Trans. Pattern Anal. Mach. Intell., vol. 29, no. 6, Jun. 2007.

[28] S. Thrun, W. Burgard, and D. Fox, "A Real-Time Algorithm for Mobile Robot Mapping with Applications to Multi-Robot and 3D Mapping," in IEEE Int. Conf. on Robotics and Automation (ICRA), vol. 1. IEEE, 2000, pp. 321-328.

[29] J.-L. Blanco, J.-A. Fernández-Madrigal, and J. González, "Efficient Probabilistic Range-Only SLAM," in IEEE/RSJ Int. Conf. Intelligent Robots and Systems (IROS). IEEE, 2008, pp. 1017-1022.

[30] L. Bruno and P. Robertson, "WiSLAM: Improving FootSLAM with WiFi,” 2011, pp. 1-10.

[31] B. Ferris, D. Fox, and N. D. Lawrence, "WiFi-SLAM using Gaussian Process Latent Variable Models," in Proc. Int. Joint Conf. on Artificial Intelligence (IJCAI), vol. 7, no. 1, Jan. 2007, pp. 2480-2485.

[32] O. J. Woodman, "An Introduction to Inertial Navigation," University of Cambridge, Computer Laboratory, Tech. Rep. UCAM-CL-TR-696, Aug. 2007.

[33] C. Jekeli, Inertial Navigation Systems with Geodetic Applications. Walter de Gruyter, 2001

[34] W. T. Fong, S. K. Ong, and a. Y. C. Nee, "Methods for In-Field User Calibration of an Inertial Measurement Unit Without External Equipment," Measurement Science and Technology, vol. 19, no. 8, 2008.

[35] N. Gordon, D. Salmond, and A. F. M. Smith, "Novel Approach to Nonlinear/Non-Gaussian Bayesian State Estimation," IEE Proc. Radar Signal Processing, vol. 140, no. 2, pp. 107-113, 1993.

[36] M. Arulampalam, S. Maskell, N. Gordon, and T. Clapp, "A Tutorial on Particle Filters for Online Nonlinear/Non-Gaussian Bayesian Tracking," IEEE Trans. Signal Process., vol. 50, no. 2, pp. 174-188, Feb. 2002.

[37] F. Gustafsson, "Particle Filter Theory and Practice with Positioning Applications," IEEE Aerosp. Electron. Syst. Mag., vol. 25, no. 7, pp. 53-82, 2010.

[38] C. Gentner, T. Jost, W. Wang, S. Zhang, A. Dammann, and U.-C. Fiebig, "Multipath Assisted Positioning with Simultaneous Localization and Mapping," IEEE Trans. Wireless Commun., vol. 15, no. 9, pp. 61046117, Sep. 2016.

[39] G. Casella and C. P. Robert, "Rao-Blackwellisation of Sampling Schemes," Biometrika, vol. 83, no. 1, pp. 81-94, 1996. 